\title{
Collapsing glomerulopathy with rare associated coxsackie virus infection: A case report
}

\author{
XUEJING ZHU, HONG LIU, SHUGUANG YUAN, XIANGQING XU, ZHEN DONG and FUYOU LIU \\ Department of Nephrology, Kidney Institute, Second Xiangya Hospital, Central South University, \\ Changsha, Hunan 410011, P.R. China
}

Received May 18, 2015; Accepted January 29, 2016

DOI: $10.3892 /$ etm.2016.3161

\begin{abstract}
A 38-year-old Chinese man was admitted to the Second Xiangya Hospital of the Central South University (Changsha, China) with heavy proteinuria and rapidly progressing renal failure with nephrotic syndrome. An initial renal biopsy identified collapsing glomerulopathy (CG) with characteristic segmental collapse of the glomerular tuft and marked hypertrophy and hyperplasia of the visceral epithelial cells. A second renal biopsy showed dilation of glomerular capillary loops as a result of effective treatment with rapamycin and anti-viral therapy. Serology for the coxsackie virus antibody was positive when the collapsing lesion was present, and became negative following treatment, which indicated a strong association between the development of CG and coxsackie virus infection. To the best of our knowledge, this is the first case report of CG associated with coxsackie virus infection.
\end{abstract}

\section{Introduction}

Collapsing glomerulopathy (CG) is a distinct clinical and pathological entity of idiopathic nephrotic syndrome, and its incidence in Asia has increased in recent years (1). The Columbia classification defines $\mathrm{CG}$ as the presence of glomerular capillary collapse in $\geq 1$ glomerulus (2). It exhibits marked hypertrophy and hyperplasia of the visceral epithelial cells, which is accompanied by tubular-interstitial injury and high resistance to immunosuppressive therapy (3).

Correspondence to: Dr Hong Liu, Department of Nephrology, Kidney Institute, Second Xiangya Hospital, Central South University, 139 Middle Renmin Road, Changsha, Hunan 410011, P.R. China

E-mail: liuh0618@163.com

Abbreviations: CG, collapsing glomerulopathy; EM, electron microscopy; CMV, cytomegalovirus

Key words: collapsing glomerulopathy, coxsackie virus, renal biopsy
The key epidemiological factors associated with $\mathrm{CG}$ include recreational drug use, hematolymphoid malignancies, autoimmune diseases and genetic mutations. CG has also been linked to infection with several types of viruses, including human immunodeficiency virus (HIV), Epstein-Barr virus (also called human herpesvirus-4), cytomegalovirus (CMV) and parvovirus B19 $(4,5)$. The present study reports a case of relapsing CG unusually associated with a coxsackie virus infection.

\section{Case report}

A 38-year-old Chinese man was admitted to the Second Xiangya Hospital of the Central South University (Changsha, China) on $22^{\text {nd }}$ January 2008 presenting with eyelid edema and anuria for 10 days. A 24-h urine protein test (cat. no. 11877801 190; Roche Diagnostics GmbH, Mannheim, Germany) result was 5.3 g. No serum HIV-1 (Intec Products, Inc., Anaheim, CA, USA), nor plasma CMV (cat. no. 3400637; Beijing Beier Biological Engineering, Co., Ltd., Beijing, China) or hepatitis B and C antibodies (Shanghai Kehua Biotech Co., Ltd., Shanghai, China), were detected, although high-titer serum coxsackie virus-specific immunoglobulin M ( $\mathrm{IgM}$; cat. no. 3400604; Beijing Beier Biological Engineering, Co., Ltd.) was observed. Serum creatinine levels were markedly increased (cat no. 77789; KANTO-PPC Inc., Shanghai, China), reaching $20.33 \mathrm{mg} / \mathrm{dl}$ and requiring hemodialysis.

Routine blood tests (Siemens Healthcare GmbH, Erlangen, Germany) detected the following: Hemoglobin, $97 \mathrm{~g} / \mathrm{l}$; total white blood cell count, $14.3 \times 10^{9}$ cells/1; neutrophils, $79.2 \%$; and lymphocytes, 6\%. Serum creatinine and albumin levels (cat no. 811RCM; Sekisui Medical, Co., Ltd., Tokyo, Japan) were $18.38 \mathrm{mg} / \mathrm{dl}$ and $23.4 \mathrm{~g} / \mathrm{l}$, respectively. A peripheral blood smear examination by light microscopy (Olympus BX51; Olympus Corporation, Tokyo, Japan) detected reactive lymphocytes. An abdominal ultrasound (SIEMENS CV70; Siemens Healthcare $\mathrm{GmbH}$ ) revealed that the right kidney measured 135x66 mm and the left 122x68 mm. Coagulation function tests detected the following: Activated partial thromboplastin time (cat. no. 557155; Siemens Healthcare $\mathrm{GmbH}$ ), $39.8 \mathrm{sec}$; fibrinogen degradation product (cat. no. 852RCM; Sekisui Medical, Co., Ltd.), $94.9 \mu \mathrm{g} / \mathrm{ml}$; D-dimer (cat.no. OPBP07; Siemens Healthcare $\mathrm{GmbH}), 46.34 \mu \mathrm{g} / \mathrm{ml}$; and fibrinogen (cat. no. B4233-27; Siemens Healthcare $\mathrm{GmbH}), 5.36 \mathrm{~g} / \mathrm{l}$. With regard to immunological 
A
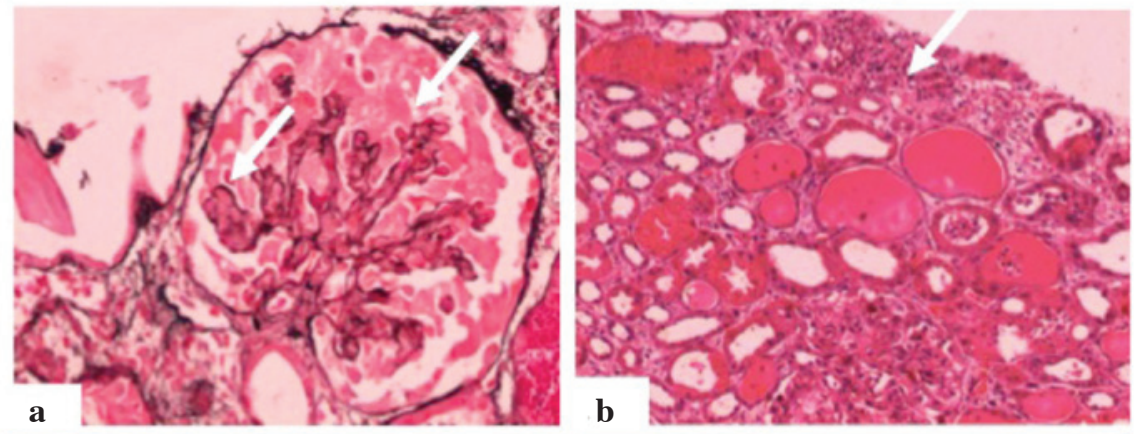

B
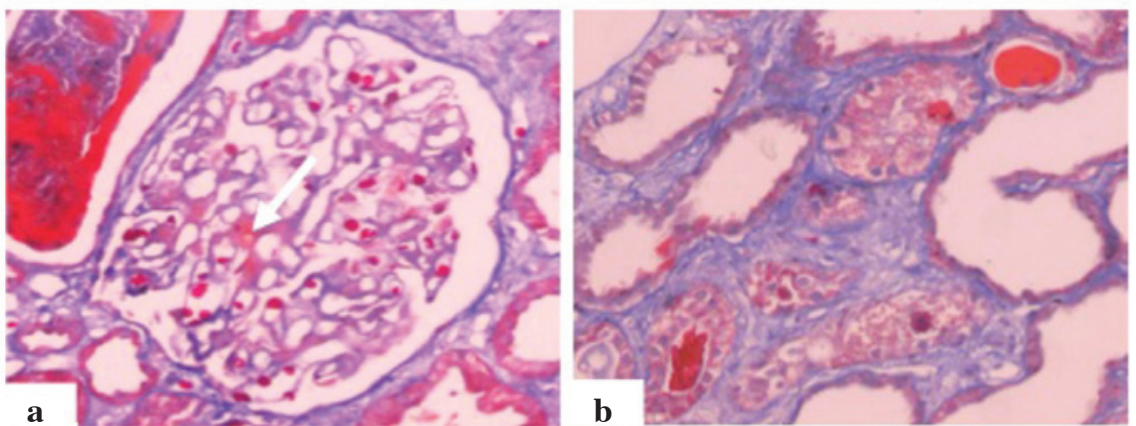

C
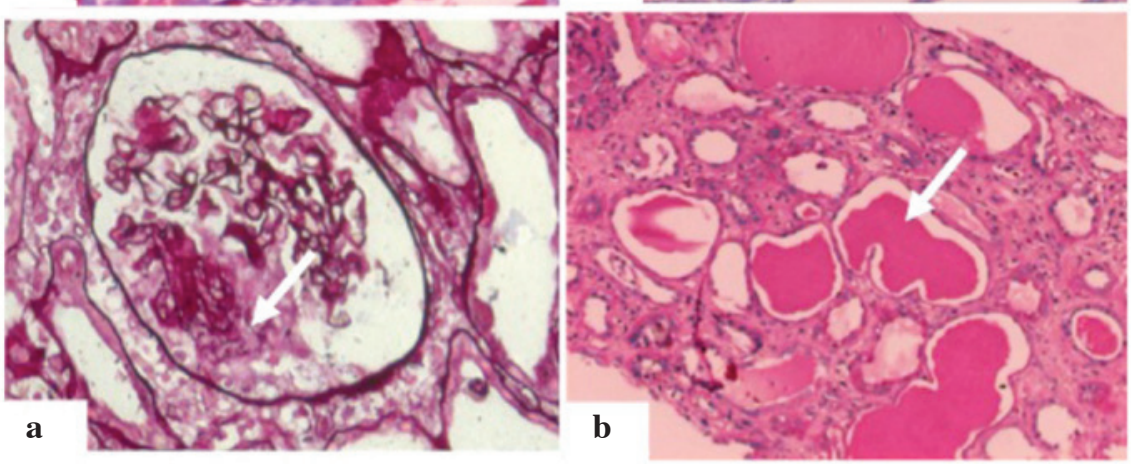

Figure 1. Renal biopsies of a 38-year-old man with collapsing glomerulopathy. (A) First renal biopsy. Light microscopy showed collapsing lesions and visceral epithelial cell proliferation with (a) vacuolar changes (PASM staining; magnification, x200; white arrow) and (b) proteinaceous casts and interstitial inflammation (hematoxylin and eosin staining; magnification, x200; white arrow). (B) Second renal biopsy, 41 days after the first. Light microscopy showed (a) mild mesangial proliferation in the glomerulus and immune deposits in the area of the mesothelium (Masson's trichrome staining; magnification, $\mathrm{x} 200$; white arrow), as well as (b) localized tubular atrophy and interstitial fibrosis (Masson's trichrome staining; magnification, x200). (C) Third renal biopsy, $\sim 3$ months after the first. Light microscopy showed (a) segmental capillary collapse with hyperplastic visceral epithelial cells with vacuoles (PASM staining; magnification, $\mathrm{x} 200$, white arrow) and (b) tubular cell flattening with massive proteinaceous casts (periodic acid-Schiff staining; magnification, x200, white arrow). PASM, periodic Schiff-methenamine.

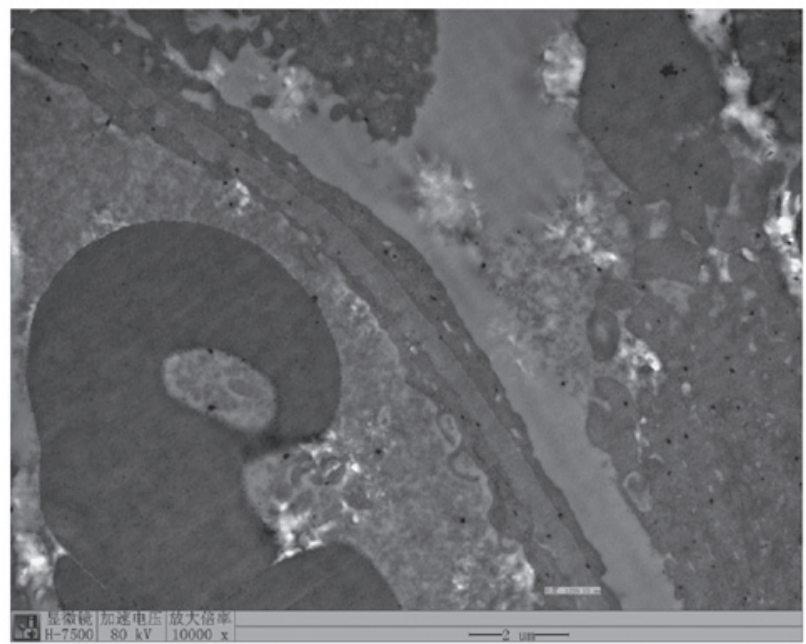

Figure 2. First renal biopsy. Electron microscopy findings showed thickening of the capillary wall, as well as diffused podocyte fusion and effacement of podocyte foot processes. parameters, the levels of complement $\mathrm{C} 3$ and $\mathrm{C} 4$ were 0.6 and $0.13 \mathrm{~g} / \mathrm{l}$, respectively (cat. nos. 446450 and 446490, respectively; Beckman Coulter, Inc., Brea, CA, USA). Antinuclear and antineutrophil cytoplasmic antibodies were negative (cat. no. 708297; Inova Diagnostics Inc., San Diego, CA, USA). No serum paraprotein, nor urinary Bence-Jones protein $\kappa$ or $\lambda$ (cat. nos. 446440 and 446470, respectively; Beckman Coulter, Inc.), were detected. Serum angiotensin-converting enzyme activity was shown to be within the normal range using a commercially available kit (cat. no. MA125290; Thermo Fisher Scientific, Inc., Waltham, MA, USA).

The patient underwent an initial renal biopsy 5 days following admission. The fresh renal tissue was fixed in $10 \%$ formaldehyde solution (Changsha Antai Fine Chemical Co., Ltd., Changsha, China), dehydrated using ethanol and turpentine oil (Hengyang Kaixin Chemical Reagent Co., Ltd., Hengyang, China) and paraffin-embedded (Leica Microsystems GmbH, Wetzlar, Germany), after which 2- $\mu \mathrm{m}$ 
sections were cut for staining with haematoxylin \& eosin (Shanghai Lanji Science and Technology Development Co., Ltd., Shanghai, China), Periodic Schiff-Methenamine Silver (Sigma-Aldrich, St. Louis, MO, USA), Masson's trichrome (Sinopharm Chemical Reagent Co., Ltd., Shanghai, China) and Periodic acid-Schiff (Sigma-Aldrich) solutions. Light microscopy (Olympus BX51) revealed that 6/9 glomeruli exhibited segmental collapse of the tuft with marked hypertrophy and hyperplasia of the visceral epithelial cells (Fig. 1A).

For immunostaining, renal tissue sections were incubated with fluorescein isothiocyanate (FITC)-conjugated rabbit anti-human complement $\mathrm{C} 3$ polyclonal antibody (1:40; F0201; Dako North America, Inc., Carpinteria, CA, USA). Immunofluorescence (IF; Olympus BX51) showed C3-positive staining in the mesangial area of glomeruli. Kidney tissue sections for electron microscopy were fixed in 2-5\% glutaraldehyde (Ted Pella, Inc., Redding, CA, USA) at $4^{\circ} \mathrm{C}$ for $1 \mathrm{~h}$ and post-fixed in $1 \%$ osmic acid (Ted Pella, Inc.) for $2 \mathrm{~h}$. The tissues were then dehydrated through graded ethanol and acetone (Tianjin Fuyu Jingxihuagong, Tianjin, China) and embedded in Epon 812 (Structure Probe Inc., West Chester, PA, USA). Electron microscopy (EM; Hitachi H7500, Hitachi, Ltd., Tokyo, Japan) revealed thickening of the capillary wall and extensive effacement of podocyte foot processes (Fig. 2). No immune deposits were found by EM. The pathological diagnosis of CG was made.

The patient was intravenously administered a high dose of anti-inflammatory therapy, including $0.25 \mathrm{~g}$ Acyclovir (Yabao Pharmaceutical Group Co., Ltd., Yuncheng, China) in $250 \mathrm{ml}$ saline, together with pulse steroids $(500 \mathrm{mg} / \mathrm{day}$ for 3 days; Paiang Pharmaceuticals Co., Ltd., Xi'an, China) and rapamycin (2 mg once daily; Pfizer, Inc., New York, NY, USA), after which hemodialysis (SWS-4000A Hemodialysis Equipment; SWS Medical, Chongqing, China) was performed. The urinary protein test yielded a negative result and serum creatinine returned to its normal levels.

The second renal biopsy was performed 41 days after the first. This time, only 1 in 16 glomeruli exhibited glomerular sclerosis (Fig. 1B). Mild proliferation of mesangial cells and focal segmental thickening were observed in the capillary walls. Capillary loops were dilated without collapse; however, IF using FITC-conjugated rabbit anti-human $\operatorname{IgM~(1:40;~}$ F0203; Dako North America, Inc.) and rabbit anti-human Clq (1:40; F0254; Dako North America, Inc.) polyclonal antibodies showed a small amount of nonspecific mesangial trapping of IgM and complement C1q trapping within the glomeruli. Serum coxsackie virus-specific IgM testing was negative. EM revealed well-formed podocyte foot processes (not shown).

On April 15, 2008, the patient presented again with fever. The 24-h urine protein test result was $5.6 \mathrm{~g}$ and the serum creatinine level had increased to $11.00 \mathrm{mg} / \mathrm{dl}$. High-level serum coxsackie virus-specific IgM was detected. A third renal biopsy was performed on April 20, 2008. The results showed pathological changes similar to those shown by the initial biopsy (Fig. 1C). Segmental or global capillary collapse and hyperplastic epithelial cells were found in all glomeruli. The tubules showed evident tubular cell flattening with massive proteinaceous casts. Immunohistochemistry was negative. The diagnosis made was that of recurrent CG.
The renal function of the patient showed no sign of improvement following treatment with Acyclovir $(0.25 \mathrm{~g}$ in $250 \mathrm{ml}$ saline), pulse steroids (500 $\mathrm{mg} /$ day for 3 days) and rapamycin (2 mg once daily). Therefore, the patient was diagnosed with end-stage renal disease (ESRD) and is currently receiving hemodialysis three times a week. Written informed consent was obtained from the patient for publication of the present study.

\section{Discussion}

The majority of coxsackie virus infections are asymptomatic; however, in a few cases such infections can be life-threatening (6). There are a limited number of case reports of any type of renal involvement in patients with coxsackie virus infection, including severe rhabdomyolysis and acute renal failure (7-9). To the best of our knowledge, this is the first case report of CG associated with coxsackie virus infection.

In the present study, coxsackie virus infection was detected in the first biopsy, but became undetectable following treatment, and was followed by recovery. However, 3 months later, serological testing for coxsackie virus antibody was positive once again, and was accompanied by the recurrence of the disease. This indicates that there is a strong association between the development of $\mathrm{CG}$ and coxsackie virus infection.

The pathogenesis of CG is not fully understood. Several studies have indicated that dysregulation of the podocyte phenotype is involved in the pathogenesis of CG $(10,11)$. Furthermore, proximal tubular epithelial cells and glomerular podocytes have been found to be highly susceptible to coxsackie virus, which thus leads to cytolysis, suggesting that coxsackie virus infections may be associated with acute and progressive renal injury (12). This supports the hypothesis that coxsackie virus infection may have been the main precipitating factor for the development of $\mathrm{CG}$ in the present patient.

$\mathrm{CG}$ is considered to be an aggressive renal disease, which usually follows a rapid course to ESRD. In a study by Conaldi et al (12), the response of CG to corticosteroids and cyclophosphamide treatment was poor. In the present case, it was demonstrated that this severe subset of FSGS could be relieved by early intervention and effective treatment with antiviral drugs, such as pulse steroid therapy and rapamycin. Following these treatments, the coxsackie virus antibody became undetectable. In addition, the second renal biopsy revealed showed relatively mild injury of glomeruli in the renal tissue of the patient, which suggests that HIV-negative CG could be less pernicious than it is considered to be, and could even be recovered under the appropriate therapy.

In conclusion, the present case of recurrent $\mathrm{CG}$ was found to be closely associated with relapsed coxsackie virus infection. Despite the recurrence of the disease, however, it is noteworthy that the patient temporarily recovered under antiviral treatment, using pulse steroids and rapamycin.

\section{Acknowledgements}

This study was supported by grants from the National Natural Science Foundation of China (grant. no. 81300600) and the Free Explore Plan of Central South University (grant no. 2012QNZT146). 


\section{References}

1. Albaqumi M and Barisoni L: Current views on collapsing glomerulopathy. J Am Soc Nephrol 19: 1276-1281, 2008.

2. D'Agati VD, Fogo AB, Bruijn JA and Jennette JC: Pathologic classification of focal segmental glomerulosclerosis: A working proposal. Am J Kidney Dis 43: 368-382, 2004.

3. Mubarak M and Kazi JI: Collapsing FSGS: A clinicopathologic study of 10 cases from Pakistan. Clin Exp Nephrol 14: 222-227, 2010.

4. Kimura T, Yasuda K, Obi Y, Satoh T, Namba T, Sasaki K, Muramoto N, Wada A, Rakugi H, Isaka Y and Hayashi T: Case of HIV-associated nephropathy accompanied by nephrotic syndrome and acute worsening of kidney function. Nihon Jinzo Gakkai Shi 54: 94-98, 2012 (In Japanese).

5. Presne C, Cordonnier C, Makdassi R, Pruna A and Fournier A Collapsing glomerulopathy and cytomegalovirus, what are the links? Presse Med 29: 1815-1817, 2000 (In French).

6. Sin J, Mangale V, Thienphrapa W, Gottlieb RA and Feuer R: Recent progress in understanding coxsackievirus replication, dissemination, and pathogenesis. Virology 484: 288-304, 2015.

7. Fodili F1, van Bommel EF: Severe rhabdomyolysis and acute renal failure following recent Coxsackie B virus infection. Neth J Med 61: 177-179, 2003.
8. Yen MH, Lee JJ, Yeh CF, Wang KC, Chiang YW, Chiang LC and Chang JS: Yakammaoto inhibited human coxsackievirus B4 (CVB4)-induced airway and renal tubular injuries by preventing viral attachment, internalization, and replication. J Ethnopharmacol 151: 1056-1063, 2014.

9. Kollios KD, Skiadopoulou E, Siondi I and Papadopoulou ZL: Coxsackie virus B1 infection in a child, complicated by severe oliguric renal failure. Clin Nephrol 67: 260-262, 2007.

10. Zhdanova O, Srivastava S, Di L, Li Z, Tchelebi L, Dworkin S, Johnstone DB, Zavadil J, Chong MM, Littman DR, et al: The inducible deletion of Drosha and microRNAs in mature podocytes results in a collapsing glomerulopathy. Kidney Int 80: 719-730, 2011.

11. Barisoni L and Nelson PJ: Collapsing glomerulopathy: An inflammatory podocytopathy? Curr Opin Nephrol Hypertens 16: 192-195, 2007.

12. Conaldi PG, Biancone L, Bottelli A, De Martino A, Camussi G and Toniolo A: Distinct pathogenic effects of group B coxsackieviruses on human glomerular and tubular kidney cells. J Virol 71: 9180-9187, 1997. 